\title{
Reflections on Authorship
}

\section{Shalini Rajaram ${ }^{1}$}

Published online: 24 July 2021

(C) Association of Gynecologic Oncologists of India 2021
Having been in academics for over 30 years, research, scientific writing and publishing become part of your life and more so for young academics. The joy of introducing to your junior colleagues, fellows and residents scientific reason, scientific inquiry, generating a scientific idea, working on developing a robust protocol, carrying out the study, analysing data and finally wrapping it up does give you immense satisfaction. This intellectual integrity with utmost standards of research is what leads to scientific excellence. Senior researchers would have gone through a similar process during their formative years with substantial contribution and intellectual inputs for every publication. In yesteryear, most papers had but 3-4 authors who significantly contributed to every aspect of the manuscript being the 'prime movers' [1]. But over the years, scientific writing has seen an uprising in the number of authors per publication (excluding multicentric trials), everyone wanting a stake in this 'publish or perish' era. How much a courtesy author has contributed is questionable, but he is cited as many times as the main author which is often unfair [2]! Authorship has become a contentious issue often leading to 'coercive authorship' and 'gift authorship'.

The inclusion of colleagues in a publication who have contributed little to the development of a research study is called 'gift authorship', 'guest authorship', 'honorary authorship', 'gratuitous authorship', 'ghost authorship', etc. [3]. What drives courtesy authorship, especially in academic institutions, has not been studied but becomes a concern when authors demand it unreasonably. Three kinds of courtesy authorship are described: gift authorship, guest authorship and coercive authorship [4]. Gift authorship is adding an author out of respect or gratitude for that individual (usually head of department, unit or institution). Guest authorship includes adding a well-known author to

Shalini Rajaram

rajaram.shalini@gmail.com

1 Department of Obstetrics and Gynecology (Gynecologic Oncology), AIIMS Rishikesh, Rishikesh, India the article to increase the visibility and value of the article. Coercive authorship consists of any kind of unjust pressures to be included as author. Each of these categories has unique implications that are likely to breach ethical principles of authorship.

The foundation of medical science is based on publishing scientific work in peer-reviewed journals and is a tool to assess the performance of anyone in academics. This publish or perish culture brings with it coercive tactics by both senior and junior academics and has led to violation authorship criteria. The individual becomes an author in a scientific article even though he or she has not made any significant contribution leave alone all 4 criteria as defined by the International Committee of Medical Journal Editors (ICMJE): (1) substantial contributions to conception or design of the work or the acquisition, analysis, or interpretation of data for the work and (2) drafting of the work or revising it critically for important intellectual content and (3) final approval of the version to be published and (4) agreement to be accountable for all aspects of the work in ensuring that questions related to the accuracy or integrity of any part of the work are appropriately investigated and resolved.

Although the practice is universally discouraged and considered academic deceit by some, the common use of courtesy authorship in academics emphasises that incentives exist. These are well known to anyone in academics, where peer-reviewed publications are 'career capital'. In academic institutions, decisions regarding promotion are linked to his or her publications and may lead to key positions, assignments, award of research funds and other academic opportunities.

Policies to diminish the practice of courtesy authorship are needed. Journal and society guidelines and ICMJE authorship criteria need to be followed to minimise inappropriate authorship. One solution is for journals and institutions to follow ICMJE authorship and non-author guidelines. Non-author contributors are defined as follows: Contributors who meet lesser than the four ICMJE criteria for authorship should not be listed as authors but 
acknowledged. Contributors who do not merit authorship may be acknowledged as 'Clinical or Participating Investigators', and their contributions should be specified. Nonauthor contributors are then included in the acknowledgments section of the manuscript [5].

Institutions could play a larger role in increasing awareness of authorship and non-author contributor roles among their faculty. The institution should have its own 'Authorship' policy on the same lines of journal policies and ICMJE criteria. This will compel the 'courtesy' and primary author to think about their role and contribution during manuscript writing and submission and not strong arm or intimidate their way into inclusion. Identifying, recognising or proving a charge of courtesy authorship is not easy because there are no objective means available as is for detecting plagiarism. Is being included as a contributor when one is not worthy actually plagiarism? [3]

Ideally, an international consensus acknowledging the role of non-author contributors will also help reduce the current practice of 'coercive' and 'gift' authorship. If promotion committees accept the value of non-author involvement towards promotion, the pressure to inappropriately include authors may be diminished. More studies and adequate control for important confounders will be required to conclusively answer this and other questions on the use of courtesy authorship [5].

Finally, institutions and particularly those in leadership positions in academia should create policies that lead to a culture of professional excellence which promote intellectual integrity. Training courses on research integrity including publication ethics and authorship issues should be integrated into the curriculum of medical students and young researchers. A large number of authors, even in highly ranked science and medical centres, are not aware of authorship guidelines and not familiar that such exist. Discussion on responsible authorship practices and guidelines should be initiated and at the institutional level $[5,6]$.

Funding None.

\section{Declarations}

Conflict of interest The authors declare that they have no conflict of interest.

Ethical approval None.

\section{References}

1. Brand RA. Further thoughts on authorship: gift authorship. Clin Orthop Relat Res. 2012;470:2926-9.

2. Moustafa K. Aberration of the citation. Acc Res. 2016;23(4):230-44.

3. Jones WJ, Mcullough LB. Is a gift authorship really a grift authorship? J Vasc Surg. 2015;61:1092-3.

4. Luiten JD, Verhemel A, Dahi Y, Luiten EJT, Gadiradj PS. Honorary authorships in surgical literature. World $J$ Surg. 2019;43:696-703.

5. McClellan MJ, Mansukhani N, Moe D, Derickson M, Chiu S, Kibbe MR, Martin MJ. Courtesy authorship in academic surgery publications. JAMA Surg. 2019;154(12):1110-6.

6. Aliukonis V, Poškute M, Gefenas E. Perish or publish dilemma: challenges to responsible authorship. Medicina (Kaunas). 2020;56(3):123. https://doi.org/10.3390/medicina56030123.

Publisher's Note Springer Nature remains neutral with regard to jurisdictional claims in published maps and institutional affiliations. 